\title{
Connecting observations of the first galaxies and the Epoch of Reionisation
}

\author{
Simon Mutch \\ University of Melbourne, Australia
}

\begin{abstract}
Dwarf galaxies are thought to be dominant contributors of ionizing photons during the Epoch of Reionisation (EoR). Our knowledge of the statistics of these high redshift galaxies is constantly improving and will take yet another important step forward with the launch of JWST. At the same time, the upper limits on the EoR $21 \mathrm{~cm}$ power spectrum are continually falling, with a firm measurement from SKA-low being a certainty in coming years. In order to maximise what we can learn from these two complimentary observational datasets, we need to be able to model them together, self-consistently. In this talk, I will present insights into the connection between galaxy formation and the EoR gained from the DRAGONS suite of semi-analytic and hydrodynamic galaxy formation simulations. Using these we find that the steep faint end slope of the high- redshift galaxy UV luminosity function extends well beyond current observational limits, indicating that only $\sim 50 \%$ of the ionising photons available for reionisation have been observed at $z<7$. I will also discuss the relative contribution of quasars to reionisation and present constraints on ionising escape fraction models.
\end{abstract}

